EP-27

\title{
The release of viral particle from hepatitis $B$ virus infected hepatocytes is inhibited by recombinant hepatitis B virus immunoglobulin (lenvervimab)
}

\author{
Kyung Chul YOON, Kwang-Woong LEE*, Sooln SEO, Seung Cheol OH, Min-Young PARK, \\ Suk Kyun HONG, Jeong-Moo LEE, Nam-Joon YI, Kyung-Suk SUH \\ Department of Surgery, Seoul National University College of Medicine, Seoul, Korea
}

Introduction: Potent and relatively low cost hepatitis B immunoglobulin (HBIG) could be good alternatives by overcoming the drawbacks of recent regimen and recombinant monoclonal HBIG (lenvervimab) has been reported the consistent avidity of variety to cloned S antigens including the immune escape mutant G145R and it did not interfere with the antibody binding in hepatitis B virus (HBV) with mutations of the $S$ gene sequence which makes drug resistance to NA. We investigated the action of intracellularly located lenvervimab in HBV infected cells which is incompletely understood yet.

Methods: We used five human hepatoma cell lines; Huh7, HepG2 as HBV negative and PLC/PRF/5, HepG2.2.15, and Hep3B expressing HBsAg. and various intracellular orgarnells markers; endosome (Rab5), autophagy marker (LC3), anti-calnexin (ER), giantin (golgi), multivesicular body (Rab7). Immunofluorescence images were used to investigate the intracellular locations of lenvervimab and HBsAg. Western blot and PEG down, ELISA were used for protein analysis.

Results: Lenvervimab was co-localized with Rab5 (early endosome marker) and and induces autophagosome but not autolysis. HBsAg and Lenvervimab were co-localized with making precipitations in cytoplasm and these precipitations were not co-localized with ER and golgi marker. Rab7 (multivesicular body marker) and lenvervimab, HBsAg were co-localized in PLC/PRF/5.

Conclusions: The release of viral particle is suppressed by lenvervimab with antigen-antibody reaction in multivesicular body. 